\title{
Archéopages
}

Archéopages Archéologie et société

40 | 04-07/2014

Villages

\section{L'eau dans les Grandes écuries de Chantilly}

Jean-Louis Bernard

\section{OpenEdition}

Journals

Édition électronique

URL : https://journals.openedition.org/archeopages/660

DOI : 10.4000/archeopages.660

ISSN : 2269-9872

\section{Éditeur}

INRAP - Institut national de recherches archéologiques préventives

\section{Édition imprimée}

Date de publication : 1 juin 2015

Pagination : $96-97$

ISSN : 1622-8545

\section{Référence électronique}

Jean-Louis Bernard, "L'eau dans les Grandes écuries de Chantilly », Archéopages [En ligne], 40 |

04-07/2014, mis en ligne le 01 janvier 2017, consulté le 22 janvier 2022. URL : http://

journals.openedition.org/archeopages/660; DOI : https://doi.org/10.4000/archeopages.660 


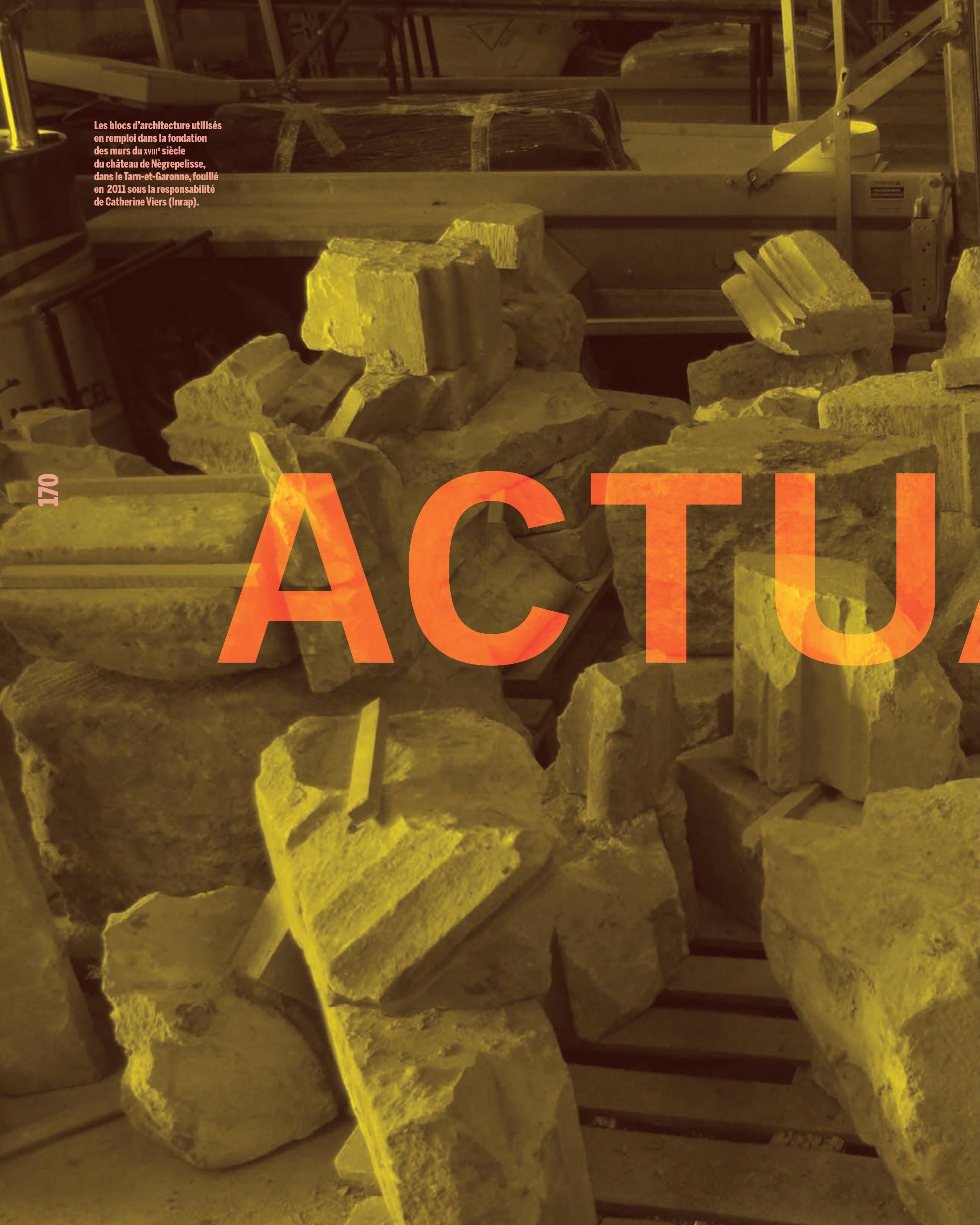




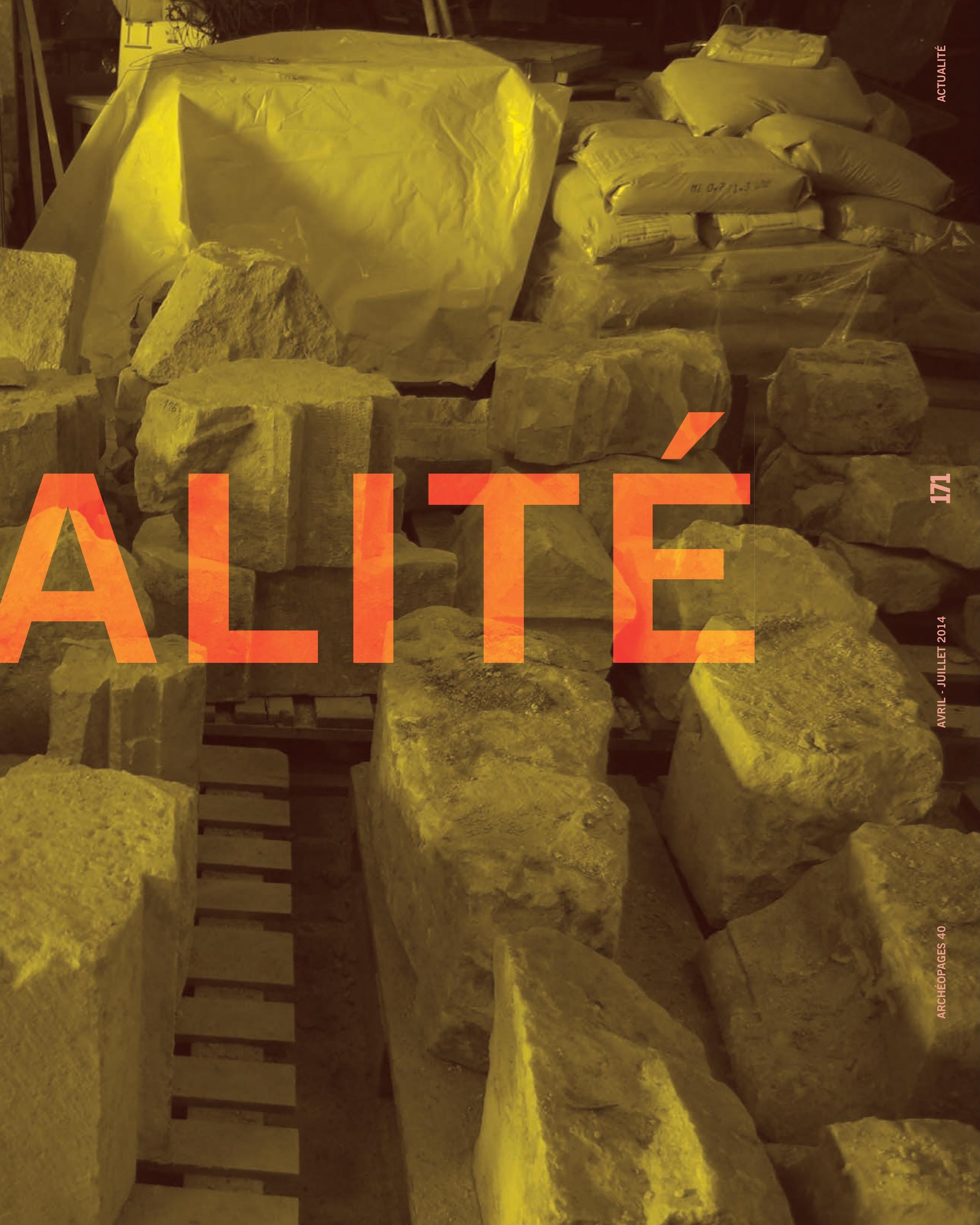




\section{Profondes mutations d'un monument}

Catherine Viers

Inrap, responsable d'opération

Site

Château

Nègrepelisse

Tarn-et-Garonne

Date

Juin 2011-janvier 2012

Superficie

$2500 \mathrm{~m}^{2}$

Équipe

Topographie

Axel Daussy, Inrap
On ne sait rien de l'origine du château de Nègrepelisse. Il apparaît en 1315, cédé par Louis X le Hutin à son oncle Louis, comte d'Évreux qui le vendit en 1318 à Pierre Duèze, seigneur de Montbrun et frère du pape Jean XX. Son fils Arnaud prit le nom de Caraman, du comté qu'il avait acheté, et le château restera en possession de cette famille jusqu'au XVII ${ }^{\mathrm{e}}$ siècle. Par voie de succession, il échoit à Henri de Beaumanoir de Lavardin en 1615 , qui le vendit à Henri de la Tour, duc de Bouillon et prince de Sédan. Après de nombreux changements de propriétaires, il revient en 1752 à Pierre-François Bergeret. Son fils, Pierre Jacques Onésime, réalisa une grande campagne de travaux de rénovation. Le château tombe en décrépitude à partir du milieu du XIX ${ }^{e}$ siècle : il sert de carrière, puis, racheté par la mairie, est arasé au niveau de la cour intérieure et aménagé en eśplanade. En 1986, la municipalité décide de mettre en valeur les vestiges de la fortification et pratique d'importants nivèlements pour dégager les murailles. À la même époque, les parements en grande partie dégarnis sont restaurés.

La fouille a concerné la totalité de l'emprise de la cour, la cave qui longe la façade nord et la tour maitresse [ill. 1]. Les niveaux de solont complètement disparu, mais plusieurs fosses ont été découvertes dans la cour, parmi lesquelles trois renferment un mobilier d'époque carolingienne attestant d'une occupation du site dès le haut Moyen Âge. La construction du château en pierre a été précédée d'une fortification, probablement en bois, dont témoigne un fossé sur lequel s'implante la courtine sud.
L'architecture du château présente les caractéristiques d'une construction des $\mathrm{XIII}^{\mathrm{e}}$-XIV ${ }^{\mathrm{e}}$ siècles : plan de type Philippe Auguste (Mesqui, 2013), moyen appareil réglé de moellons à tête dressée dans un calcaire froid éclaté au taillant, évacuation des eaux usées intégrées dans les maçonneries avec bouches au nu des parements se déversant au pied des courtines, poterne située à mi-hauteur de la courtine nord. Excepté cette dernière, l'arasement de la construction n'a permis la conservation d'aucune ouverture, ni même des fondations des murs intérieurs du château, si bien que sa disposition initiale est conjecturelle. Seule une série d'ouvertures à ébrasement simple, mais dont les pierres de parement ont disparu, atteste de la présence de jours en fente ou d'archères le long de la courtine orientale. L'examen des maçonneries a permis de mettre en évidence des reprises de chantier et deux repentir en fondation (un sur la courtine orientale et l'autre au niveau de l'articulation avec la tour maîtresse). Létude du bâti de la cave montre que celle-ci est entièrement d'époque moderne. L'ancrage du
1. Plan phasé du château. Le château de Nègrepelisse se présente sous la forme d'une enceinte quadrangulaire de $50 \mathrm{~m}$ de côté flanquée de tours rondes aux angles et est bâti sur une légère éminence en rive gauche de l'Aveyron. La tour maîtresse, de plan carré, s'implante à cheval sur la courtine méridionale.

Fosses carolingiennes

Fossé d'une fortification antérieure au XIV ${ }^{\mathrm{e}}$ siècle

Château XIV ${ }^{\mathrm{e}}$ siècle

Cave moderne (XVII ${ }^{e}$ siècle)

Restructuration du XVIII ${ }^{\mathrm{e}}$ siècle

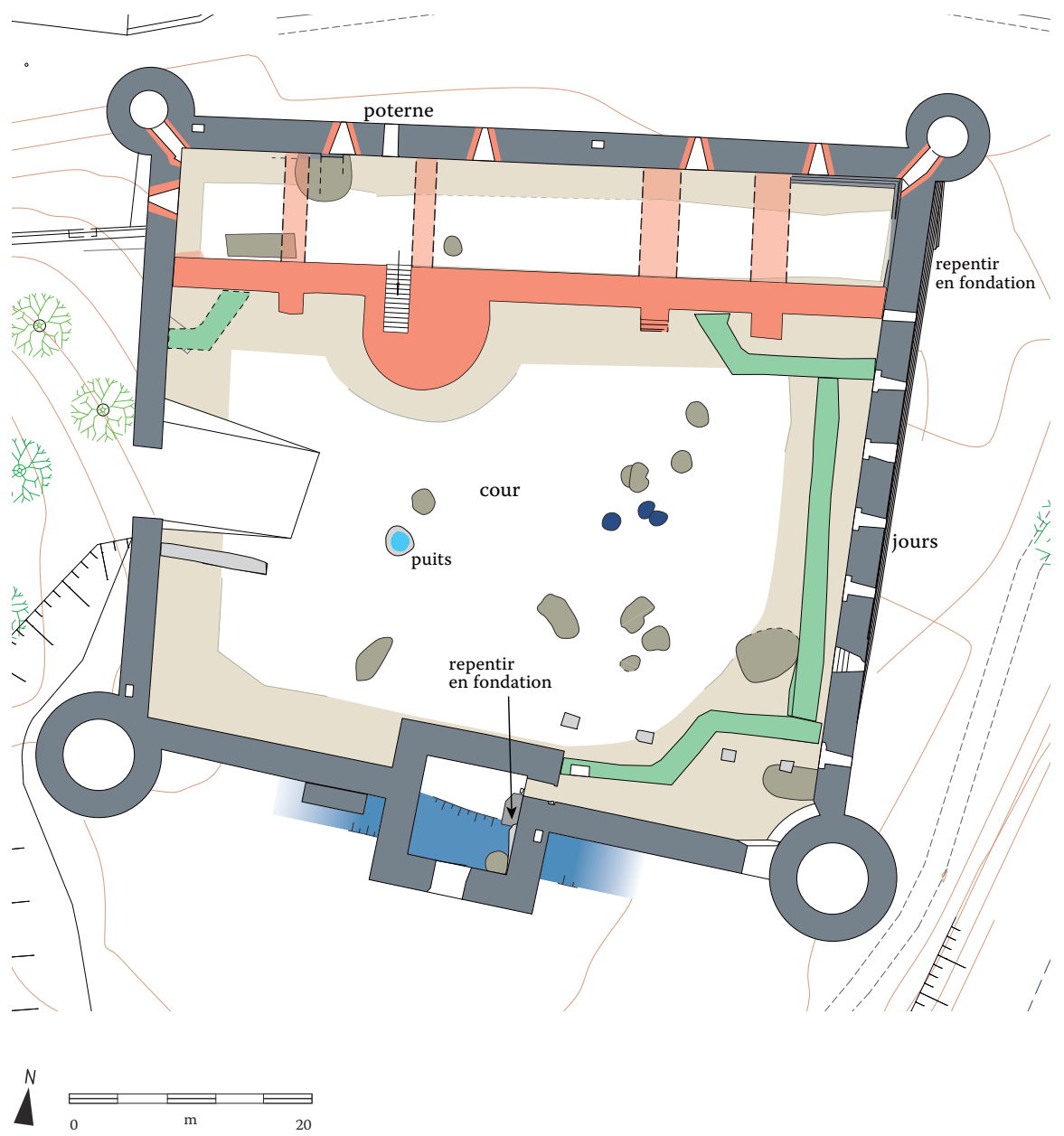



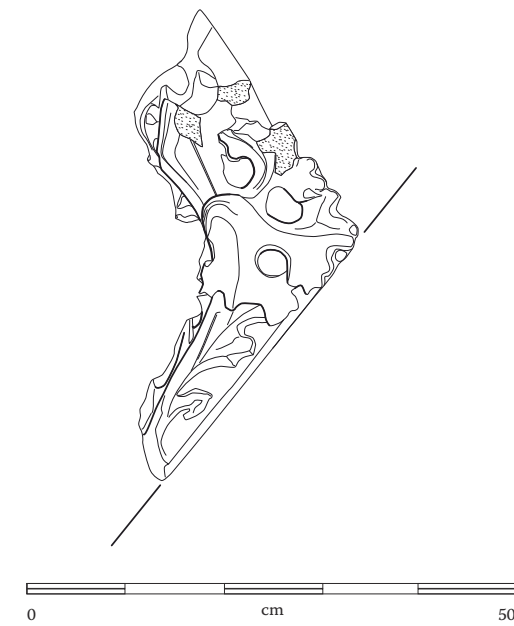

2. Crochet en feuilles de choux ornant le gâble d'un portail, découvert en huit exemplaires.

3. Proposition de restitution

d'une fenêtre à croisée à

décor de colonnettes

torsadées et bordée de

pilastres surmontés de

pinacles.

4. Proposition de restitution

d'un fronton de lucarne avec

d'un fronton de lucarne avec
épi de faîtage polygonal.

5. Restitution d'un dais, dont

la couverture pyramidale

sculptée de feuilles de

chardons et de pinacles

abritait probablement des

statues.

voûtement de cette dernière s'est fait en dégarnissant le parement médiéval de la courtine. Les travaux du XVIII ${ }^{\mathrm{e}}$ siècle transforment profondément le château. Le corps de logis au-dessus de la cave est modernisé et la cour entièrement restructurée. Des cages d'escalier et un mur écran, bâti en avant de la courtine orientale, transforment sa géométrie au goût en usage sous Louis XIV. Le démontage sous suivi archéologique de ces murs a livré un lot très homogène de plus de 230 blocs d'architecture datés entre 1460 et 1520 , utilisés en remploi dans la fondation [ill. ouverture]. Les blocs d'une même baie sont souvent regroupés dans la fondation suggérant un approvisionnement sur place. Cette découverte illustre une période du château totalement méconnue qui affirme une magnificence dont la région MidiPyrénées a gardé peu de témoignages.

Bien qu'aucune baie n'ait pu être reconstituée en totalité, le classement typologique a permis d'identifier des fragments de gâbles à accolade,
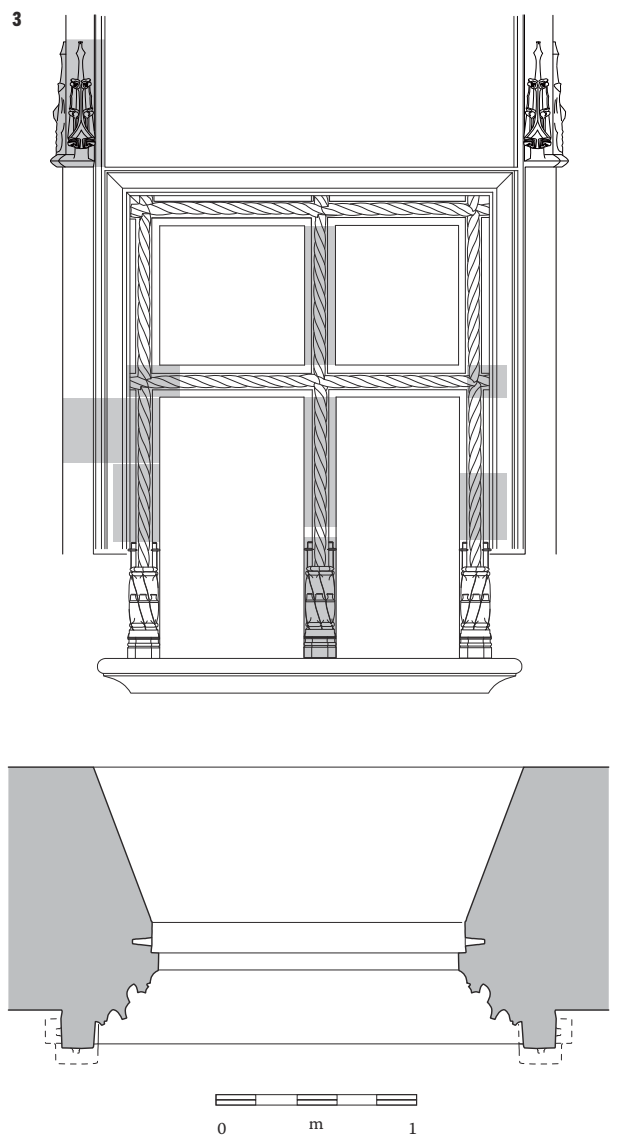

enrichis de crochets végétaux [ill. 2], ornant le sommet d'au moins deux portes. Un linteau mouluré avec cavets d'adoucissement appartient certainement à l'une d'elles. Sept types de fenêtres à croisée ont été répertoriés. La plus dépouillée comporte un simple chanfrein. La plus monumentale développe une mouluration complexe ornée de colonnettes torsadées à bases prismatiques et bordée de pilastres lisses dont le sommet est agrémenté de pinacles [ill. 3]. Les pilastres devaient filer sur toute la hauteur de la façade, encadrant les fenêtres superposées des différents niveaux. Une autre fenêtre, très incomplète, s'orne de colonnettes à décor d'écailles. Le sommet des murs gouttereaux s'agrémentait de lucarnes aux frontons en pierre dont les rampants moulurés ont été reconnus. Deux types ont été distingués, le plus imposant est surmonté d'un épi polygonal, et des mortaises permettent l'ancrage d'un décor sculpté [ill. 4]. Grâce aux entablements, au moins trois cheminées monumentales aux moulures complexes et puissantes, dont le plan alterne courbes et contre-courbes, ont été restituées. La présence de deux niches à dais [ill. 5], et de fausses gargouilles figurant des lions assis complètent l'image d'une façade très richement ornée, entièrement effacée par les transformations de la fin de l'époque moderne. L'histoire du château de Nègrepelisse, dont seules les fondations nous sont parvenues, apparaît comme celle d'un bâtiment en perpétuelle mutation, dont l'apparence, au gré du contexte politique et des modes, évolue de façon radicale : la totale transformation de l'aspect du château, au XviII ${ }^{\mathrm{e}}$ siècle; la transformation d'un édifice militaire en résidence de prestige et expression d'un pouvoir ostentatoire à la fin du Moyen Âge (Mesqui, 1993 ; Cocula, Combet, 2011). Sans la réutilisation de ce lapidaire, relégué en fondation, sa redécouverte et son étude, c'est tout un pan de cette histoire qui nous échappait.
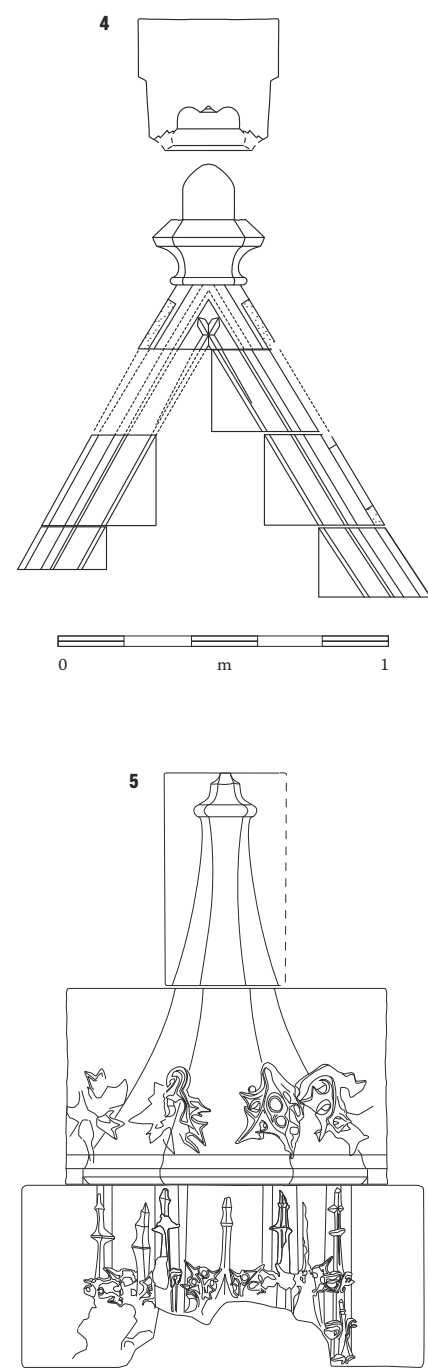

ก
Références bibliographiques

Cocula A.-M., Сомвет M., 2011: Château, naissance et métamorphoses, Actes des Rencontres d'archéologie et d'histoire en Périgord, 24-26 septembre 2010, Pessac, Bordeaux Ausonius (coll. Scripta mediaevalia, 19).

MESQUI J., 1993: Châteaux et enceintes de la France médiévale. De la défense à la résidence. t. 2, De la défense à la résidence, Paris, éd. Picard.

Mesqui J., 2013 : Châteaux et enceintes de la France médiévale. De la défense à la résidence. t. 1, Les organes de la défense, Paris, éd. Picard. 